\title{
To the Sun and beyond
}

Tell Bartolo Luque and Fernando Ballesteros how far the Sun is from the Earth, and they will tell you the size of the Universe.

T

he measures of the heavens and the Earth have been inextricably intertwined throughout history. Until the seventeenth century, determining geographical position required knowledge of the position and the distance to celestial bodies, measures that in turn depended on the distance between two observing sites on Earth. For example, the first determination of the Earth's perimeter ${ }^{1}$, carried out by Eratosthenes of Cyrene (276-194 вс), crucially depended on the actual distance between the cities of Alexandria and Syene. Precision geography was not possible without astronomy or vice versa, and their measurements were always relative to other measurements. Aristarchus of Samos (310-230 вс) concluded that the distance to the Sun ${ }^{2}$ - roughly the astronomical unit was 19.1 times larger than the distance to the Moon; a distance that, in turn, he calculated as a function of the Earth's diameter.

Nearly two millennia would have to pass before the next significant step forward was taken by Johannes Kepler (1571-1630). His third law ${ }^{3}$ on planetary motion states that, for every planet, the ratio between the cube of the semi-major axis of its elliptical orbit and the square of its orbital period is a constant. This allowed the distance to the Sun of a planet to be calculated from the planet's period, but as a multiple of the Earth-Sun distance.

The way out of this vicious circle moving from a system of relative magnitudes to absolute ones would arrive with the precise determination of the average distance from the Earth to the Sun, known as the astronomical unit $(\mathrm{au})$.

The first absolute measurements of the au were enabled by the invention of the telescope, which allowed Christiaan Huygens (16291695) in 1659 to make precise measurements of the angular distance between the Sun and Venus ${ }^{4}$. Reusing ideas from Aristarchus and assuming that the diameter of Venus is equal to Earth's, he estimated that the au was $157,125,000 \mathrm{~km}\left(\right.$ ref. $\left.^{5}\right)$ - exceeding our current estimate by $5 \%$. Thirteen years later, Giovanni Domenico Cassini (1625-1712) and Jean Richer (1630-1696) simultaneously

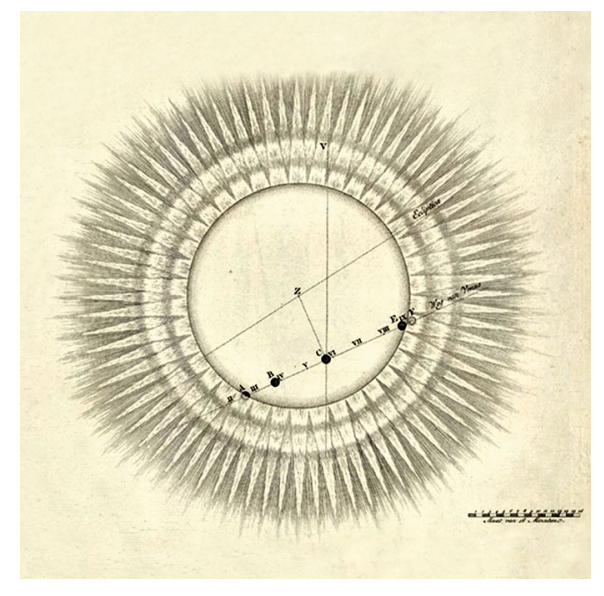

Credit: Science History Images / Alamy Stock Photo

observed the position of Mars with respect to the stars from France and French Guiana, estimating that the au was equal to $141,500,000 \mathrm{~km}\left(\right.$ (ref. $\left.^{5}\right)-5 \%$ lower than the current estimate. The net was being tightened.

The telescope also allowed the observation of transits - tiny solar eclipses of the inner planets Venus and Mercury. In 1716, Edmond Halley (1656-1742) proposed a method ${ }^{6}$ to measure the au more accurately by observing transits of Venus from two remote locations. Since the observation should be diurnal, it was not possible to measure the parallaxes of Venus and of the Sun with respect to the stars. The ingenious trick of Halley's method was that it allowed the real solar parallax to be found from the apparent parallax of Venus with respect to the Sun. Then, knowing the distance between the locations, one can determine the distance to the Sun.

During the Venus transits of 1761 (pictured) and 1769, astronomers from around the world spread across the planet to take more than 200 observations. After analysing all the data, in 1771, Joseph Jérôme Lefrançois de Lalande (1732-1807) provided the most accurate measurement of the au at that time: the Sun was 153,000,000 km away, which exceeds by $2 \%$ the current estimation of $149,597,870.7 \mathrm{~km}$ obtained by telemetry and radar $^{5}$. Once the au was determined, the true dimensions of the Solar System could finally be revealed.

By the same token, in 1838 Friedrich Bessel (1784-1846) determined the first stellar parallax $^{7}$ of 61 Cygni, supposedly one of the closest stars to Earth. From observations from opposite extremes of the Earth's orbit, Bessel concluded a parallax of 0.314 arcseconds (") and realized that the Universe was much larger than previously imagined; the au fell short for stellar distances. Such measurements of parallaxes are obtained naturally in parsecs (pc), where $1 \mathrm{pc}$ is the distance to be moved away so that a segment of 1 au subtends an angle of 1"; consequently, $1 \mathrm{pc}=1 \mathrm{au} / \tan (1 ")$ $\approx 206,264.8$ au by definition. Therefore, the absolute length of the parsec depends on the value of the au.

When converting his angular measurements of the star 61 Cygni to absolute distances, Bessel realized that the light from the star took 10.3 years to arrive, and in the process indirectly invented the most popular measure today of interstellar distances: the light year. Now, the Universe's diameter is believed to be 93 billion light years - at least the observable bit. The precise determination of the au opened a new window that allowed us to appreciate the enormous size of the Universe.

Bartolo Luque ${ }^{1}$ and

Fernando J. Ballesteros (D)2

${ }^{1}$ Universidad Politécnica de Madrid, Madrid, Spain.

${ }^{2}$ Observatori Astronòmic, Universitat de València,

Paterna, Spain.

e-mail: bartolome.luque@upm.es;

fernando.ballesteros@uv.es

Published online: 2 December 2019

https://doi.org/10.1038/s41567-019-0685-3

References

1. Cleomedes On the Circular Motions of the Celestial Bodies (circa $50 \mathrm{BC}$ ).

2. Aristarchus of Samos On the Sizes and Distances (of the Sun and Moon) (circa 270 вс).

3. Kepler, J. Harmonices Mundi Book 5, Ch. 3, 189 (1619).

4. Goldstein, S. J. Jr Observatory 105, 32-33 (1985).

5. Beatty, J. K., Collins Petersen, C. \& Chaikin, A. (eds) The New Solar System (Cambridge University Press, 1999).

6. Halley, E. Phil. Trans. R. Soc. 29, 454-464 (1716).

7. Bessel, F. W. Astron. Nach. 16, 65-96 (1838).

$\begin{array}{lll}\mathrm{m} & \AA \mathrm{s} & \mathrm{u}\end{array} E_{\mathrm{h}}$

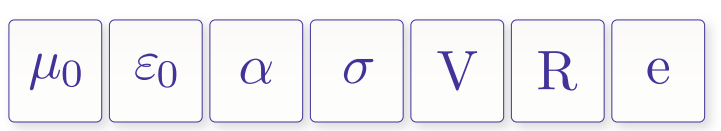

